\title{
An Evaluation Model for Tailings Storage Facilities Using Improved Neural Networks and Fuzzy Mathematics
}

\author{
Sen Tian ${ }^{1,2}$ and Jianhong Chen $^{1,2}$ \\ ${ }^{1}$ School of Resources and Safety Engineering, Central South University, Changsha 410083, China \\ ${ }^{2}$ The Deep Metal Mineral Exploitation and Hazard Control Key Laboratory, Changsha 410083, China \\ Correspondence should be addressed to Jianhong Chen; 115501019@csu.edu.cn
}

Received 2 May 2014; Revised 5 September 2014; Accepted 7 September 2014; Published 12 November 2014

Academic Editor: Fuzhen Xuan

Copyright (c) 2014 S. Tian and J. Chen. This is an open access article distributed under the Creative Commons Attribution License, which permits unrestricted use, distribution, and reproduction in any medium, provided the original work is properly cited.

\begin{abstract}
With the development of mine industry, tailings storage facility (TSF), as the important facility of mining, has attracted increasing attention for its safety problems. However, the problems of low accuracy and slow operation rate often occur in current TSF safety evaluation models. This paper establishes a reasonable TSF safety evaluation index system and puts forward a new TSF safety evaluation model by combining the theories for the analytic hierarchy process (AHP) and improved back-propagation (BP) neural network algorithm. The varying proportions of cross validation were calculated, demonstrating that this method has better evaluation performance with higher learning efficiency and faster convergence speed and avoids the oscillation in the training process in traditional BP neural network method and other primary neural network methods. The entire analysis shows the combination of the two methods increases the accuracy and reliability of the safety evaluation, and it can be well applied in the TSF safety evaluation.
\end{abstract}

\section{Introduction}

According to Dixon-Hardy and Engels [1], safety problem caused by tailings has become one of the most serious problems in mine engineering. TSFs, as the man-made debris flow source of danger with high potential energy, cause a waste of resources, the loss of life and property, and environmental pollution due to current limited production technology and equipment, as well as safety awareness [2,3]. With the industrial development, the number of TSFs has reached more than 12,000 [4]; therefore, the research on it has academic value but also economic and social benefits. The stability analysis of the TSFs thus is indispensable for the research on mining [4].

TSFs are used for piling up tailings and other industrial waste residues, which can be usually divided into five systems, including tailings storage system, flood control system, water return system, transportation system, and safety management system. This paper established a reasonable TSF safety evaluation index system, which includes an exhaustive list of seventeen evaluation indexes, and assessed the unambiguous prioritisation of influence for the general objective based on the AHP methodology with a case study.

Furthermore, several evaluation indexes in this system were chosen as the input vectors of the improved BP neural network (BPNN) to build a new TSF safety evaluation model by adopting a variable learning rate, introducing the backpropagation mechanism, improving the adjustment rate of weights, and accelerating the convergence speed of the error functions.

According to the simulation experiments, this improved BPNN algorithm has better evaluation performance with higher learning efficiency and faster convergence speed and avoids the oscillation in the training process of traditional BPNN algorithm. The entire analysis shows the combination of improved BPNN algorithm and fuzzy AHP methodology increases the accuracy and reliability of the safety evaluation, and it can be well applied in the TSF safety evaluation. 


\section{Background}

Many domestic and overseas researchers have done researches on the TSF safety problems. Yin et al. [4] introduced the stability analysis model of a Chinese TSF; Rico et al. [5] presented the urgent need for European regulations regarding technical standards of tailings disposal; Wei et al. [2] analyzed the current situation, shortcomings, and key weaknesses, as well as future development trends for tailings storage facilities; Hu and Liu [6] introduced the security monitoring system of a tailings dam. In the case of environmental problems caused by TSFs, Romero et al. [7] studied the solidphase control on the mobility of potentially toxic elements in an abandoned lead/zinc mine tailings impoundment.

In the case of previous research, stability analysis on TSFs has been developed. Grangeia et al. [8] made the analysis for assessing, preventing, mitigating, and controlling risks of the TSF; Wang et al. [9] introduced the safety assessment of the tailings pond with a case study; Yin et al. [10] made the experimental study on the mechanism effect of seepage on microstructure of tailings experiment; Sun et al. [11] presented a new tailings dam flood overtopping failure evolution pattern and so forth.

However, previous studies mainly focus on the prevention of environment pollution instead of the inherent stability of TSFs. Furthermore, the accuracy of analysis is limited by qualitative evaluation to a large extent. Generally, this kind of qualitative evaluation plays a role in prevention of TSF accidents indeed, however, it's easy to overlook hidden dangers only through on-site inspection. In view of the drawbacks in the current TSF safety evaluation methods, this paper presented a more comprehensive approach for the complicated safety evaluation to improve precision and reliability.

\section{Methodology}

\subsection{BPNN Algorithm}

3.1.1. Network Frame. The traditional BPNN frame [12-14] often consists of input layer, hidden layer, and output layer. It sometimes contains more than one hidden layer; the outputs of each layer are sent directly to each neuron of the next layer [15]; it also may contain a bias neuron that produces constant outputs but receives no inputs. If the frame includes only one hidden layer, it is called one hidden layer or three-layer BP neural network frame, which is shown in Figure 1.

In Figure $1,\left(x_{1}, x_{2}, \ldots, x_{N 1}\right)$ is expressed as the input vector of the input layer; $\left(z_{1}, z_{2}, \ldots, z_{N 3}\right)$ is the output vector of output layer; $\left(t_{1}, t_{2}, \ldots, t_{N 3}\right)$ is the expected output vector, $\left(\Delta_{1}, \Delta_{2}, \ldots, \Delta_{N 3}\right)$ are described as the errors between actual output vector and anticipated one, $\left(w_{i 1}, w_{i 2}, \ldots, w_{i h}, w_{N 1 N 2}\right)$ is the connection weight vector between input layer and hidden layer, and $\left(w_{h 1}, w_{h 2}, \ldots, w_{h j}, w_{N 2 N 3}\right)$ is the one between hidden layer and output layer.

According to the activation function of hidden layer, the derivation value of each neuron can be calculated by the input

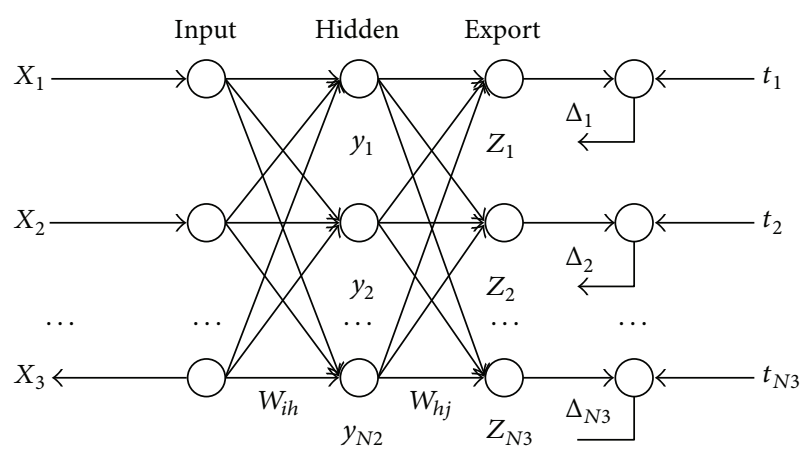

FIgURE 1: Three-layer BP neural network frame.

value and the connection weight between input layer and hidden layer, as expressed below:

$$
\begin{gathered}
y_{j}=f\left(\mathrm{Net}_{j}\right), \quad j=1,2, \ldots, N_{2}, \\
\mathrm{Net}_{j}=\sum_{j=0}^{N_{1}} w_{i j} x_{i}, \quad i=1,2, \ldots, N_{1} .
\end{gathered}
$$

Likewise, the neurons derivation value of output layer can be calculated from

$$
\begin{aligned}
& Z_{k}=f\left(\operatorname{Net}_{k}\right), \quad k=1,2, \ldots, N_{3}, \\
& \operatorname{Net}_{k}=\sum_{j=0}^{N_{3}} w_{j k} y_{j}, \quad j=1,2, \ldots, N_{2} .
\end{aligned}
$$

The expression of global error is given as [15]

$$
E=\sum_{k=1}^{N_{3}} \Delta_{k}=\frac{1}{2 N_{1}} \sum_{t=1}^{N_{1}} \sum_{k=1}^{N_{3}}\left(T_{k}-Z_{k}\right)^{2} .
$$

3.1.2. BPNN Algorithm Implementation. After the network frame is determined, the prepared sample data should be trained in the network with the following training steps [16, 17].

(1) Initialization of Network Training Parameters. BPNN needed scale standardization. Initialize the connection weight between each neuron within the interval $[-1,1]$ and preset the allowable training error $e$, learning rate $r$, and maximum iteration number $N$. The input data $X=\left\{x_{1}, x_{2}, \ldots\right.$, $\left.x_{N 1}\right\}$ for training and its corresponding anticipative output data $T=\left\{t_{1}, t_{2}, \ldots, t_{N 3}\right\}$ are selected randomly and put into the input layer.

(2) Forward-Propagating Calculation of the Network. The output value of the hidden layer is solved with Sigmoid activation function $f(x)$, input vector $X$, and the connection weight $w_{i h}$; the output vector $Z=\left\{Z_{1}, Z_{2}, \ldots, Z_{N_{3}}\right\}$ is then calculated in terms of the hidden layer output value $y$ and the connection weight $w_{h j}$. The total error $E$ is obtained by error function; the allowable error is calculated with the actual 
output value and the anticipative one; if $E<e$, the training ends; otherwise, go to step (3).

(3) Adjustment of Back-Propagation Network Error. The weight allowance $\Delta w_{h j}$ between output and hidden layer and $\Delta w_{\text {ih }}$ between input and hidden layer can be calculated with weighting allowance function and total error $E$. Then $\Delta w_{h j}$ and $\Delta w_{i h}$ should be adjusted with the following formulas:

$$
\begin{aligned}
& w_{h j}^{\prime}=w_{h j}+\Delta w_{h j}, \\
& w_{i h}^{\prime}=w_{i h}+\Delta w_{i h} .
\end{aligned}
$$

(4) Recalculation of Forward-Propagating of the Network. The output value $Z$ and total error $E$ are calculated with adjusted connection weights $w_{h j}^{\prime}$ and $w_{i h}^{\prime}$. If $E<e$, the network training ends; otherwise, return to step (3) until $E<e$, or the iteration number reaches $N$.

\subsection{The Optimization of BPNN Algorithm}

3.2.1. Optimization of Convergence Rate. BPNN is based on solid and rigorous theory derivation; however, it was also found that there were many limitations in the training process, including slow convergence rate, the emergence of local extremum, and limitation of practical application. In view of these drawbacks, the improved BP algorithm was proposed for using the variable learning rate, adjusting the connection weights between different nodes dynamically, improving the convergence rate in the training process, and so on [18]. Figure 2 shows the propagation mechanism of improved BP algorithm. The algorithm implementation procedure of the improved BPNN is described in detail as follows.

The threshold of the network can be adjusted with the weights and added into the weight matrix [19]. Suppose $U$ is expressed as the connection weight matrix between input layer and hidden layer, $V$ is the connection weight matrix between hidden layer and output layer, $X$ is the input vector, $Y$ is the output vector of the network, $M$ is the sum of samples, $f(x)$ is the activation function, and $d_{y}$ is the anticipated output value. Then,

$$
\begin{aligned}
X & =\left[\begin{array}{ccccc}
1 & x_{11} & x_{12} & \ldots & x_{1 I} \\
1 & x_{21} & x_{22} & \ldots & x_{2 I} \\
1 & x_{31} & x_{32} & \ldots & x_{3 I} \\
\ldots & \ldots & \ldots & \ldots & \ldots \\
1 & x_{M 1} & x_{M 2} & \ldots & x_{M I}
\end{array}\right] \\
& =\left(X_{m i}\right)_{M \times(I+1)}, \\
U & =\left[\begin{array}{cccc}
u_{01} & u_{02} & \ldots & u_{0 J} \\
u_{11} & u_{12} & \ldots & u_{1 J} \\
u_{21} & u_{22} & \ldots & u_{2 J} \\
\ldots & \ldots & \ldots & \ldots \\
u_{I 1} & u_{I 2} & \ldots & u_{I J}
\end{array}\right] \\
& =\left(u_{i j}\right)_{J \times(I+1)},
\end{aligned}
$$

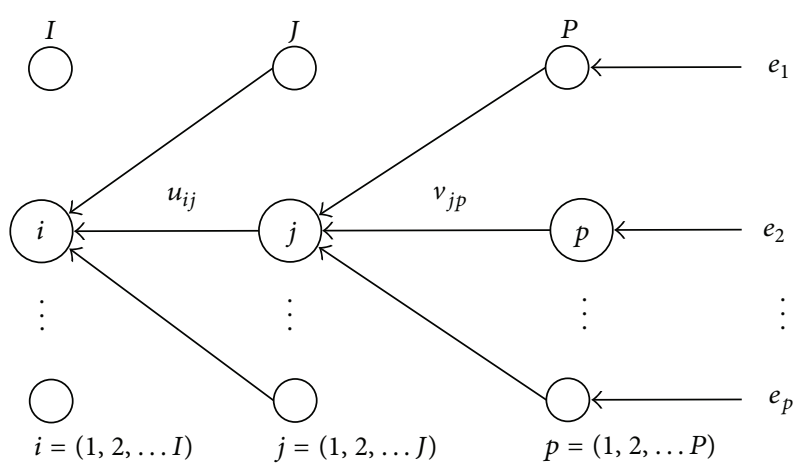

FIGURE 2: Improved BP algorithm propagation mechanism.

$$
\begin{aligned}
V & =\left[\begin{array}{cccc}
v_{01} & v_{02} & \ldots & v_{0 P} \\
v_{11} & v_{12} & \ldots & v_{1 P} \\
v_{21} & v_{22} & \ldots & v_{2 P} \\
\ldots & \ldots & \ldots & \ldots \\
v_{J 1} & v_{J 2} & \ldots & v_{J P}
\end{array}\right] \\
& =\left(v_{j p}\right)_{P \times(J+1)} .
\end{aligned}
$$

The forward-propagation process of improved BPNN with the innovative adaptive learning rate algorithm is similar to the process of the traditional one [20]:

$$
\begin{gathered}
f(x)=\left(1+e^{-\alpha x}\right)^{-1}, \\
Y_{j}=f\left(\sum_{i=1}^{I+1} x_{m i} u_{i j}\right), \\
Y_{p}=f\left(\sum_{j=1}^{J+1} Y_{j} v_{j p}\right), \\
E(n)=\frac{1}{2} \sum_{p=1}^{P}\left(d_{y}-Y_{p}\right)^{2} .
\end{gathered}
$$

The general ideas of the weight adjustment are shown as follows [21, 22].

(1) Suppose rs $U$ is the row spread matrix of matrix $U, r s V$ is the row spread matrix of matrix $V$, and

$$
\begin{aligned}
& U=\left(u_{i j}\right)_{(I+1) \times J}, \\
& V=\left(v_{j p}\right)_{(J+1) \times P},
\end{aligned}
$$

$\mathrm{rs} \mathrm{U}$

$$
=\left[u_{01}, u_{02}, \ldots, u_{0 J}, u_{11}, u_{12}, \ldots, u_{1 j}, \ldots, u_{I 1}, u_{I 2}, \ldots, u_{I J}\right],
$$

$\mathrm{rsV}$

$$
=\left[v_{01}, v_{02}, \ldots, v_{0 P}, v_{11}, v_{12}, \ldots, v_{1 P}, \ldots, v_{J 1}, v_{J 2}, \ldots, v_{J P}\right] .
$$


(2) Supposing $W$ is the whole weight matrix of the BPNN, $W=[\mathrm{rs} U, \mathrm{rs} V]$. To easily finish the backpropagation calculation, we define $W^{*}$ as the reversed order weight matrix of $W, W^{*}=[\mathrm{rs} V, \mathrm{rs} U]$. If $m$ and $n$ are the row vector numbers of the whole weight matrix, then there exists a relationship:

$$
W^{*}=[\mathrm{rs} V, \mathrm{rs} U]=\left[w_{1}, w_{2}, \ldots, w_{n}, \ldots, w_{m}\right] \text {, }
$$

where $m=(I+1) \times J+(J+1) \times P, n=(J+1) \times P$.

(3) The weights of $W^{*}$ can be denoted as $w_{i}, i \in[1, m]$. If $W(n)$ is the weight matrix of BPNN after learning $n$ times and $W^{*}(n)$ is the reversed order weight matrix, then the initial weight matrix and initial reversed order weight matrix can be expressed as $W(0)$ and $W^{*}(0)$. When adjusting the weights of $W^{*}, w_{i}(n)$ is the value of $w_{i}$ at the $n$th learning.

In the forward-propagation stage, the learning termination condition of $W(n)$ is

$$
E(n) \leq e
$$

Here, $E(n)$ is the error in the $n$th learning and $e$ is the preset precision.

If it does not satisfy the termination condition, the learning continues to enter the stage of back-propagation error adjustment.

To classify the process of back-propagation of the new algorithm, we take any weight $w_{i}$ in $W^{*}$; for example, the adjustment process is shown below:

(1) If the gradient of $w_{i}$ equals zero, $\partial E / \partial w_{i}=0$, then we adjust the next weight $w_{i+1}$; if $\partial E / \partial w_{i} \neq 0$, the weights are calculated by (10) in the $n$th learning:

$$
\begin{gathered}
\Delta w_{i}(n)=-\eta_{i}(n) \frac{\partial E}{\partial w_{i}}, \\
w_{i}(n+1)=w_{i}(n)+\Delta w_{i}(n) .
\end{gathered}
$$

(2) In the forward-propagation stage, if $w_{i}(n+1)$ is superior to $w_{i}(n)$, namely, the error decreases, then,

$$
\eta_{i}(n) \times 2=\eta_{i}(n) .
$$

Along with the negative gradient direction of $w_{i}(n)$, $w_{i}(n+1)$ is recalculated with new learning rate $\eta_{i}(n)$. Then, a new weight $w_{i}^{(1)}(n+1)$ is obtained; if $w_{i}^{(1)}(n+$ $1)$ is superior to $w_{i}(n+1)$, then the learning rate is increased with (14). By analogy, if $w_{i}^{(2)}(n+1)$ is still superior to $w_{i}^{(1)}(n+1)$, the learning rate will be increased again. If $w_{i}^{(m)}(n+1)$ after $m+1$ times calculations is not equal to $w_{i}^{(m-1)}(n+1)$, then,

$$
\begin{gathered}
w_{i}(n+1)=w_{i}^{(m-1)}(n+1), \\
\eta_{i}(n)=\eta_{i}^{(m-1)}(n) .
\end{gathered}
$$

(3) Suppose $w_{i}(n+1)$ is calculated along with forwardpropagation direction and inferior to $w_{i}(n)$; the error increases; then,

$$
\frac{\eta_{i}(n)}{2}=\eta_{i}(n)
$$

Along with the negative gradient direction of $w_{i}(n)$, $w_{i}(n+1)$ is recalculated with the new learning rate $\eta_{i}(n)$. If new $w_{i}(n+1)$ is inferior to $w_{i}(n)$, then we decrease the learning rate $\eta_{i}(n)$ again with (14) until $w_{i}(n+1)$ is superior to $w_{i}(n)$ as long as $\partial E / \partial w_{i} \neq 0$.

In fact, when learning rate was lower, training time got longer and convergence became slower. When learning rate was too high, oscillation and divergence had emerged; this caused an unstable system. After the complex adjustment process, a new weight matrix $W(n+1)$ and a new reversed order weight matrix $W^{*}(n+1)$ are acquired. $W(n+1)$ is the optimal weight matrix of this network if the termination condition (9) is met; otherwise, $W^{*}(n+1)$ should be put into a new round of adjustment until the termination condition (9) is met.

3.2.2. The Improved BPNN Algorithm Implementation. The application of improved BPNN with a new adaptive learning rate algorithm should be implemented step by step from the algorithm derivation to the computer programming. Following are its general training steps [23].

(1) The initialization of BP neural network: set up the network structure and confirm the expected input and output of sample. Randomly select the minor weight matrix $W$ and vector $\eta$ and set the error precision $e$ of the network learning.

(2) Input sample with forward-propagation: record the forward-propagating error of $E^{(0)}$ this time.

(3) Counterpropagation: adjust the weight. For weight matrix $W^{*}=\left[w_{1}, w_{2}, \ldots, w_{n}, \ldots, w_{m}\right]$ with reversed order, select vector quantity $\Delta w_{i}(n), i=1,2, \ldots, m$ to adjust the elements in it one by one. If the gradient weight of error function is less than the preset minimum gradient $\varepsilon$, that is, $\partial E / \partial w_{i}<\varepsilon$, then switch to step (5). If the gradient weight is larger than the preset minimum gradient $\varepsilon$, that is, $\partial E / \partial w_{i}>\varepsilon$, then switch to step (4).

(4) Seeking the optimum weight in the gradient direction: adjust the weight according to (12) and calculate error $E^{(1)}$ with forward-propagation. Compare $E^{(1)}$ and $E^{(0)}$. If network error decreases, then increase the learning rate, readjust the weight, and calculate error $E^{(1)}$ with forward-propagation until the error no longer decreases. If network error increases, then decrease the learning rate, readjust the weight, and calculate error $E^{(1)}$ with forward-propagation until the error no longer decreases.

(5) Forward-propagation with new weight: update the network value $E^{(1)}$. If the error is less than the preset 


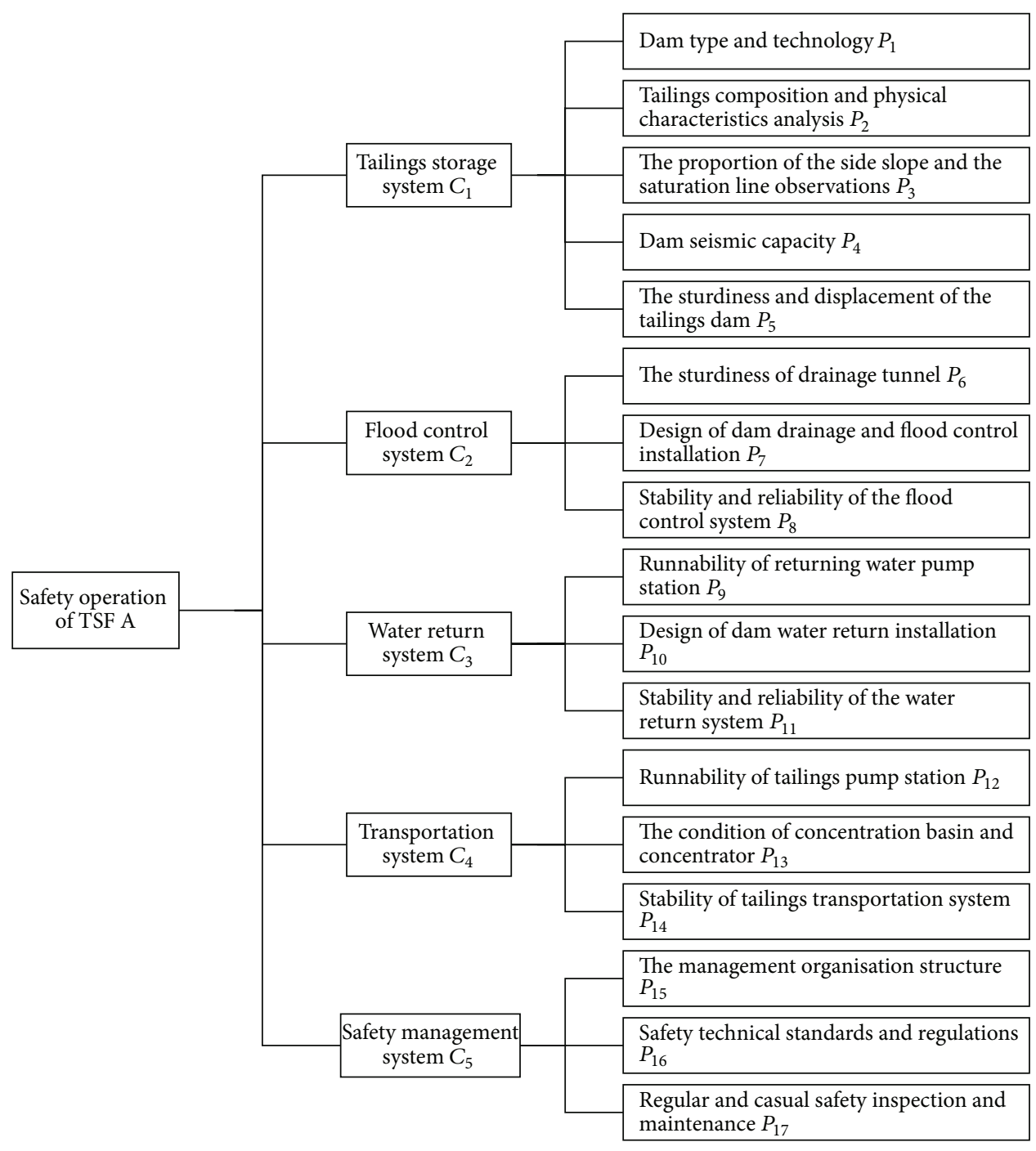

FIGURE 3: Evaluation structure for the safe operation of the TSF.

precision $e$, then the network jumps out of the whole propagation; stop the learning and turn to step (6). If the error is larger than the preset precision $e$, make $i=i+1$. When $i<m$, which means that there is also some weight of node that has not been adjusted, then turn to step (2) and add a weight adjustment. When $i=m$, which means that all of weight of node has been adjusted but the error at that time could not meet the accuracy requirement, make $i=0$, a new round of iterative learning from the first node.

(6) Finish the BP neural network learning and record the final weight matrix.

\section{Theory and Calculation}

4.1. Establishment of Evaluation Index System. TSF safe operation is affected by multiple aspects, including natural factors, dam type and technology, management, and environment. Statistics show that TSF failure modes include four types, foundation failure, structure failure, overtopping failure, and seepage failure; we analyzed the risk factors of these failure modes and established the evaluation index system in Figure 3.

4.2. AHP Procedures. The fuzzy AHP methodology is effective for sectors of risk assessment and has been successfully applied in many fields [24-27]. In this paper, we analyzed the safety situation of a typical TSF case in China based on fuzzy AHP methodology.

We selected several experts' perspectives and suggestions from our research organisation, assessed the evaluation indexes in this system according to the investigation reports and the regional inspections, and finally ranked them by the prioritisation of influence for the general objective of this system [28]. The total sorting for the hierarchy structure is shown in Table 1.

Our results indicate that each judgment matrix has a satisfactory consistency because each CR is less than 0.10 [24]. 
TABLE 1: Total sorting for the hierarchy structure.

\begin{tabular}{llc}
\hline Evaluation indexes & Weight & Sorting \\
\hline Dam type and technology $P_{1}$ & 0.0299 & 9 \\
Tailings composition and physical characteristics analysis $P_{2}$ & 0.0093 & 15 \\
The proportion of the side slope and the saturation line observations $P_{3}$ & 0.1169 & $\mathbf{3}$ \\
Dam seismic capacity $P_{4}$ & 0.0708 & 0.0129 \\
The sturdiness and displacement of the tailings dam $P_{5}$ & 0.0325 & 0.1631 \\
The sturdiness of drainage tunnel $P_{6}$ & 0.2726 \\
Design of dam drainage and flood control installation $P_{7}$ & 0.0199 \\
Stability and reliability of the flood control system $P_{8}$ & 0.0562 \\
Runnability of returning water pump station $P_{9}$ & 0.1057 \\
Design of dam water return installation $P_{10}$ & 0.0292 \\
Stability and reliability of the water return system $P_{11}$ & 0.0035 \\
Runnability of tailings pump station $P_{12}$ & 0.0116 \\
The condition of concentration basin and concentrator $P_{13}$ & $\mathbf{2}$ \\
Stability of tailings transportation system $P_{14}$ & 11 \\
The management organisation structure $P_{15}$ & $\mathbf{1}$ \\
Safety technical standards and regulations $P_{16}$ & $\mathbf{4}$ \\
Regular and casual safety inspection and maintenance $P_{17}$ & 10 \\
\hline
\end{tabular}

Overall, the most risky index is the stability and reliability of the flood control system; it may depend on the design of dam drainage and flood control installation, which is also the second most risky index. The third most risky index, the proportion of the side slope and the saturation line observations, may cause an increase in dam slumping due to pipe and dam slope seepage. The fourth most risky index is the stability and reliability of the water return system, which should be emphasised because it will directly affect flood control system and even the entire safe operation of TSFs. In addition, dam seismic capacity, regular and casual safety inspection and maintenance, and the sturdiness of drainage tunnel are also important in this example. Actually, no evaluation index in this system should be neglected because the indexes may interact with each other. Therefore, relevant precautions should be taken timely by the order of prioritisation and emergency to avoid accidents and dangers.

On the basis of the total sorting, the top five evaluation indexes are chosen as the input vectors $x_{1}$ to $x_{5}$ of the improved BP neural network to build a new TSF safety evaluation model, namely, stability and reliability of the flood control system $x_{1}$, design of dam drainage and flood control installation $x_{2}$, the proportion of the side slope and the saturation line observations $x_{3}$, stability and reliability of the water return system $x_{4}$, and dam seismic capacity $x_{5}$.

\subsection{Preparation of Samples (See [29])}

4.3.1. Training Sample. TSF safety evaluation is a pattern recognition problem in essence. We compared the measured values of a group or several groups of TSF safety risk factors with the standard values and analyzed the closest safety evaluation level of measured values, and that was the recognition result of BPNN model. Based on improved BP algorithm principles, this paper integrated the safety posture grade of
TSFs to construct the training samples for the network with chosen indexes. In order to improve them, this paper adopted the section interpolation method to extend the training sample set. Through the comparative analysis of the linear interpolation and random interpolation, the network model trained by the extended samples proved to be more stable. Here, we selected three typical China's typical TSF examples in Hunan province and obtained 63 training samples to form the training samples set for our research according to the investigation reports and the regional inspections of all indexes in this model from year 2009 to 2013, and a total of 78 samples were chosen in this model.

4.3.2. Test Sample. We selected 5 groups of monitoring data consisting of 15 testing samples. In order to obtain the simulation results more accurately, the cross validations were calculated; that is, 15 testing samples were chosen randomly from 78 total samples for several times until each sample was tested once.

4.4. Determination of BP Network Topology. The settings of network topology include the number of network layers and hidden layers, nodes number of the input layer, the output layer, and the hidden layer. The reasonability of the setting directly relates to the precision and objectivity of the evaluation and the application value of this model [23].

4.4.1. Network Layer. As described before, if the hidden layer is solved with Sigmoid function and the activation function between input layer and output layer is a linear function, the multilayer forward neural network with single hidden layer is approximate to rational function with any precision. It is not difficult to get the conclusion that the training effect is easily reached by increasing the nodes number of hidden 


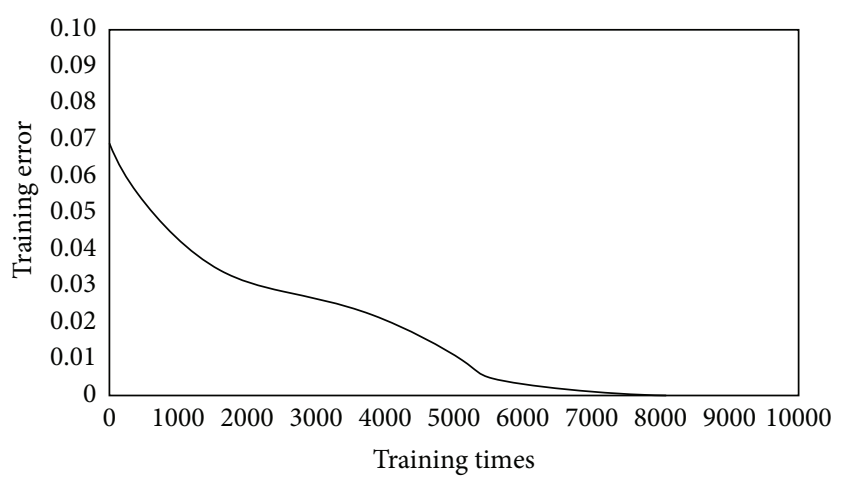

FIGURE 4: Improved BP algorithm training error.

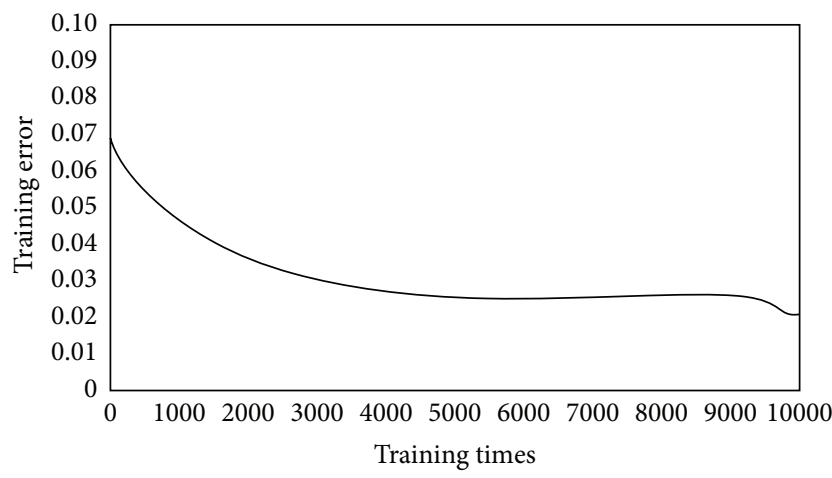

FIGURE 5: Traditional BP algorithm training error.

layer to improve the network precision and decrease the error. Therefore, a three-layer network model was built in this paper.

4.4.2. Nodes Number. The nodes number of input and output layer is mainly determined by the practical situation in the research. Here, we chose five nodes for input layer corresponding to five chosen indexes, as well as one node for output layer corresponding to recognition result of the TSF safety evaluation. Finally, ten optimal hidden layer nodes were chosen with golden cut method.

\section{Simulation Experiments Analysis and Results}

5.1. Performance Analysis of the Improved Algorithm. In order to verify the effectiveness of this improved model, this paper made the numerical experiments to compare the actual result of improved BPNN and traditional one [30, 31].

In our study, MATLAB 7.0 was used to realize the neural network model and the algorithm by using nonlinear approximation property of neural network to deal with this complex nonlinear function.

The nonlinear function is constructed as

$$
y=\sin (x), \quad x=[0,2 \pi] .
$$

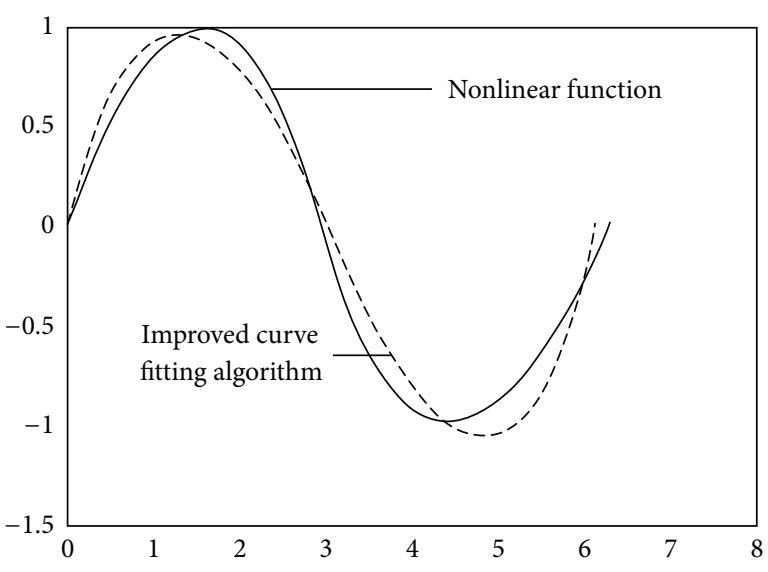

FIGURE 6: Improved BP algorithm fitting curve.

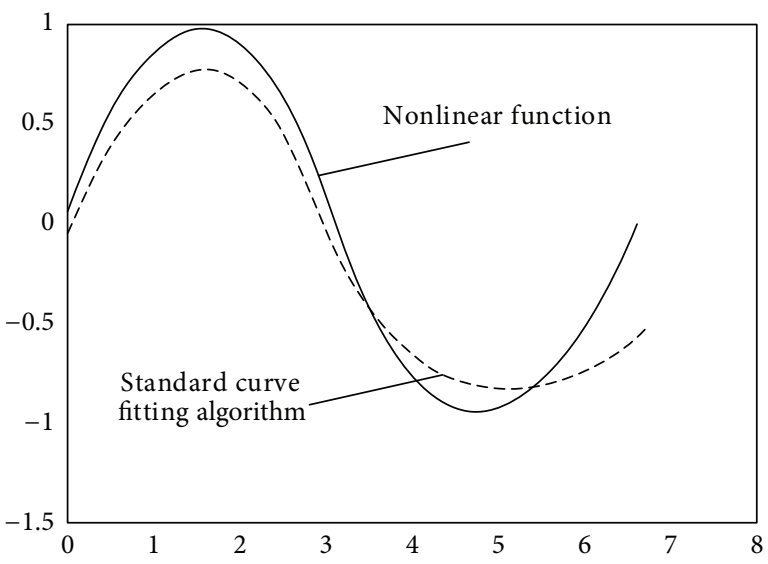

FIGURE 7: Traditional BP algorithm fitting curve.

Two models with ten nodes in the hidden layer and 63 training samples were simulated at the same experiment environment. Sigmoid function is expressed as transfer function of hidden layer and output layer; we defined anticipated training precision as 0.0001 and maximum operation number as 10000 . The training errors of both training models are shown in Figures 4 and 5, together with the fitting curves in Figures 6 and 7.

From the figures above, we can analyze that the improved BPNN algorithm makes the learning of network weights more efficient, optimizes the convergence speed, and avoids the oscillation of the traditional BPNN algorithm. Experiment results confirm the feasibility of TSF safety evaluation model proposed in this paper.

5.2. Results and Discussion. We introduced the simulation experiments into real example for the TSF safety evaluation model. Here, danger coefficient is the target value, usually divided into four levels [12]: $0.1,0.2,0.3$, and 0.4 ; they correspond to the four result grades: safe, defective, seriously defective, and extremely dangerous. 
TABLE 2: The result of the improved BP algorithm.

\begin{tabular}{cccc}
\hline SN & Expert evaluation & Algorithm evaluation & Dangerous index \\
\hline 1 & 0.3 & 0.3 & $x_{1}, x_{2}$ \\
2 & 0.4 & 0.4 & $x_{2}, x_{3}, x_{4}$ \\
3 & 0.1 & 0.1 & \\
4 & 0.2 & 0.2 & $x_{2}$ \\
5 & 0.4 & 0.4 & $x_{1}, x_{3}, x_{4}$ \\
\hline
\end{tabular}

TABLE 3: The result of comparison of algorithms.

\begin{tabular}{lcc}
\hline Methods & $\begin{array}{c}\text { Average } \\
\text { accuracy (\%) }\end{array}$ & $\begin{array}{c}\text { Average operation } \\
\text { time (s) }\end{array}$ \\
\hline $\begin{array}{l}\text { Fuzzy comprehensive } \\
\text { evaluation method }\end{array}$ & 71 & 1026 \\
RBFNN algorithm & 68.9 & 183 \\
GRNN algorithm & 84.2 & 132 \\
EIMNN algorithm & 81.5 & 158 \\
Traditional BP & 79.8 & 164 \\
algorithm & 96.1 & 45 \\
$\begin{array}{l}\text { Improved BP } \\
\text { algorithm }\end{array}$ & & \\
\hline
\end{tabular}

Among them, expert evaluation was obtained by the investigation reports and the regional inspections. From Table 2, it can be seen that the results of our evaluation model and expert evaluation are equivalent, and this algorithm has proved that it can accurately calculate the dangerous indexes.

To well evaluate the application ability of the safety evaluation model proposed in this paper, we compared its accuracy and operation time with fuzzy comprehensive evaluation method and other primary neural network methods, as shown in Table 3.

While RBFNN is radial basis function neural network, EIMNN is Elman neural network and GRNN refers to general regression neural network.

To sum up, this improved safety evaluation model has better performance than other primary neural network methods and fuzzy comprehensive evaluation method, whether in accuracy or in operation time.

\section{Conclusion}

This paper presented a new safety evaluation approach to assess the safe operation of TSF; simulation experiments verified that the proposed algorithm evaluation is accurate and efficient and can be well applied in engineering practice.

First, this paper established a reasonable TSF safety evaluation index system, which includes an exhaustive list of seventeen evaluation indexes and assessed the unambiguous prioritisation of influence for the general objective based on the AHP methodology with a case study. Next, on the basis of the AHP total sorting, several evaluation indexes were chosen as the input vectors of the improved BPNN algorithm to build a new TSF safety evaluation model; it optimized the convergence speed, adopted the back-propagation mechanism to decrease the algorithm errors, and avoided the oscillation in the training process of traditional BPNN algorithm. The varying proportions of cross validations were calculated; this improved algorithm proved to have higher accuracy and faster convergence speed, according to several simulation experiments and comparisons with other primary neural network methods and fuzzy comprehensive evaluation method.

Through an efficient combination of improved BPNN and fuzzy AHP methodology using qualitative and quantitative analyses, this paper presents a more comprehensive approach for the complicated safety evaluation to improve precision, accuracy, and reliability.

\section{Conflict of Interests}

The authors declare that there is no conflict of interests regarding the publication of this paper.

\section{Acknowledgments}

This work is supported by the project fund of the National Natural Science Foundation of China (no. 50774092) and the National Outstanding Doctoral Dissertations Special Fund (no. 200449).

\section{References}

[1] B. W. Dixon-Hardy and J. M. Engels, "Guidelines and recommendations for the safe operation of tailings management facilities," Environmental Engineering Science, vol. 24, no. 5, pp. 625-637, 2007.

[2] Z. Wei, G. Yin, J. G. Wang, L. Wan, and G. Li, "Design, construction and management of tailings storage facilities for surface disposal in China: case studies of failures," Waste Management and Research, vol. 31, no. 1, pp. 106-112, 2013.

[3] M. Rico, G. Benito, and A. Díez-Herrero, "Floods from tailings dam failures," Journal of Hazardous Materials, vol. 154, no. 1-3, pp. 79-87, 2008.

[4] G. Yin, G. Li, Z. Wei, L. Wan, G. Shui, and X. Jing, "Stability analysis of a copper tailings dam via laboratory model tests: a Chinese case study," Minerals Engineering, vol. 24, no. 2, pp. 122130, 2011.

[5] M. Rico, G. Benito, A. R. Salgueiro, A. Díez-Herrero, and H. G. Pereira, "Reported tailings dam failures: a review of the European incidents in the worldwide context," Journal of Hazardous Materials, vol. 152, no. 2, pp. 846-852, 2008.

[6] J. Hu and X. Z. Liu, "Design and implementation of tailings dam security monitoring system," Procedia Engineering, vol. 26, pp. 1914-1921, 2011.

[7] F. M. Romero, M. A. Armienta, and G. González-Hernández, "Solid-phase control on the mobility of potentially toxic elements in an abandoned lead/zinc mine tailings impoundment, Taxco, Mexico," Applied Geochemistry, vol. 22, no. 1, pp. 109-127, 2007.

[8] C. Grangeia, P. Ávila, M. Matias, and E. F. da Silva, "Mine tailings integrated investigations: the case of Rio tailings (Panasqueira Mine, Central Portugal)," Engineering Geology, vol. 123, no. 4, pp. 359-372, 2011. 
[9] T. Wang, Y. Zhou, Q. Lv, Y. Zhu, and C. Jiang, "A safety assessment of the new Xiangyun phosphogypsum tailings pond," Minerals Engineering, vol. 24, no. 10, pp. 1084-1090, 2011.

[10] G. Yin, Q. Zhang, W. Wang, Y. Chen, W. Geng, and H. Liu, "Experimental study on the mechanism effect of seepage on microstructure of tailings," Safety Science, vol. 50, no. 4, pp. 792796, 2012.

[11] E. J. Sun, X. K. Zhang, Z. X. Li, and Y. H. Wang, "Tailings dam flood overtopping failure evolution pattern," Procedia Engineering, vol. 28, pp. 356-362, 2012.

[12] J. Kang and W. J. Meng, "System identification based on improved BP neural networks," Journal of Computational Information Systems, vol. 8, no. 5, pp. 2099-2106, 2012.

[13] W. Wu, J. Wang, M. Cheng, and Z. Li, "Convergence analysis of online gradient method for BP neural networks," Neural Networks, vol. 24, no. 1, pp. 91-98, 2011.

[14] Z. H. Tang and R. J. Li, "A novel data mining classification method based on neural network," Journal of Computational Information Systems, vol. 6, no. 4, pp. 1069-1076, 2010.

[15] H. X. Yang, C. Zhao, X. J. Du, and J. Wang, "Risk prediction of city distribution engineering based on BP," Systems Engineering Procedia, vol. 5, pp. 55-60, 2012.

[16] L. Zhang, J. Luo, and S. Yang, "Forecasting box office revenue of movies with BP neural network," Expert Systems with Applications, vol. 36, no. 3, pp. 6580-6587, 2009.

[17] Q. M. Wu and M. Wei, "A mathematical expression for air ESD current waveform using BP neural network," Journal of Electrostatics, vol. 71, no. 2, pp. 125-129, 2013.

[18] Z. Zhou, "Target identification based on Error back propagation neural network and fuzzy support vector machines," Journal of Computational Information Systems, vol. 7, no. 5, pp. 1663-1671, 2011.

[19] D. Zheng, P. Peng, and S. Fan, "A research of dynamic compensation of coriolis mass flowmeter based on BP neural networks," Instruments and Experimental Techniques, vol. 56, no. 3, pp. 365-370, 2013.

[20] C. H. Tang, Y. Xie, B. H. Qiang, X. Wang, and R. Zhang, "Security situation prediction based on dynamic BP neural with covariance," Procedia Engineering, vol. 15, pp. 3313-3317, 2011.

[21] Z.-H. Guo, J. Wu, H.-Y. Lu, and J.-Z. Wang, "A case study on a hybrid wind speed forecasting method using BP neural network," Knowledge-Based Systems, vol. 24, no. 7, pp. 10481056, 2011.

[22] Y. Tan, G. Zeng, and L. Yu, "Energy analysis for an executable program on a single computer based on BP neural network," Journal of Experimental and Theoretical Artificial Intelligence, vol. 25, no. 1, pp. 105-117, 2013.

[23] G. Jing, W. Du, and Y. Guo, "Studies on prediction of separation percent in electrodialysis process via BP neural networks and improved BP algorithms," Desalination, vol. 291, pp. 78-93, 2012.

[24] A. C. Caputo, P. M. Pelagagge, and P. Salini, "AHP-based methodology for selecting safety devices of industrial machinery," Safety Science, vol. 53, pp. 202-218, 2013.

[25] H. A. Nefeslioglu, E. A. Sezer, C. Gokceoglu, and Z. Ayas, "A modified analytical hierarchy process (M-AHP) approach for decision support systems in natural hazard assessments," Computers and Geosciences, vol. 59, pp. 1-8, 2013.

[26] O. S. Vaidya and S. Kumar, "Analytic hierarchy process: an overview of applications," European Journal of Operational Research, vol. 169, no. 1, pp. 1-29, 2006.
[27] G. Z. Zheng, N. Zhu, Z. Tian, Y. Chen, and B. H. Sun, "Application of a trapezoidal fuzzy AHP method for work safety evaluation and early warning rating of hot and humid environments," Safety Science, vol. 50, no. 2, pp. 228-239, 2012.

[28] S. Lee, W. Kim, Y. M. Kim, and K. J. Oh, "Using AHP to determine intangible priority factors for technology transfer adoption," Expert Systems with Applications, vol. 39, no. 7, pp. 63886395, 2012.

[29] S. Ding, C. Su, and J. Yu, "An optimizing BP neural network algorithm based on genetic algorithm," Artificial Intelligence Review, vol. 36, no. 2, pp. 153-162, 2011.

[30] H. S. Wang, Y. N. Wang, and Y. C. Wang, "Cost estimation of plastic injection molding parts through integration of PSO and BP neural network," Expert Systems with Applications, vol. 40, no. 2, pp. 418-428, 2013.

[31] L. Song, X. Qu, and S. Ye, "Improved SFS 3D measurement based on BP neural network," Image and Vision Computing, vol. 25 , no. 5, pp. 614-622, 2007. 


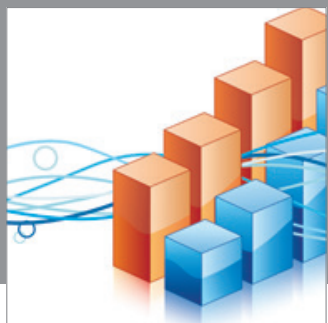

Advances in

Operations Research

mansans

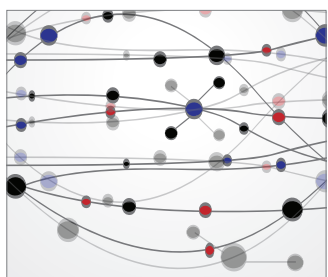

The Scientific World Journal
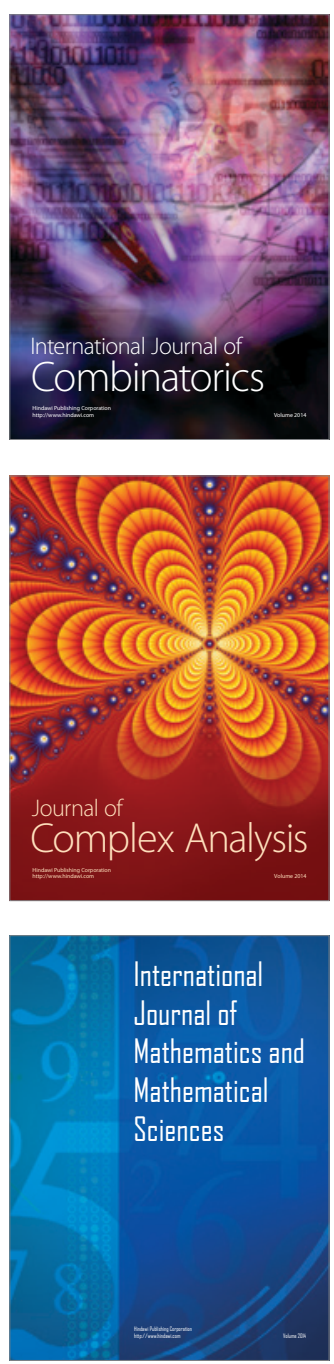
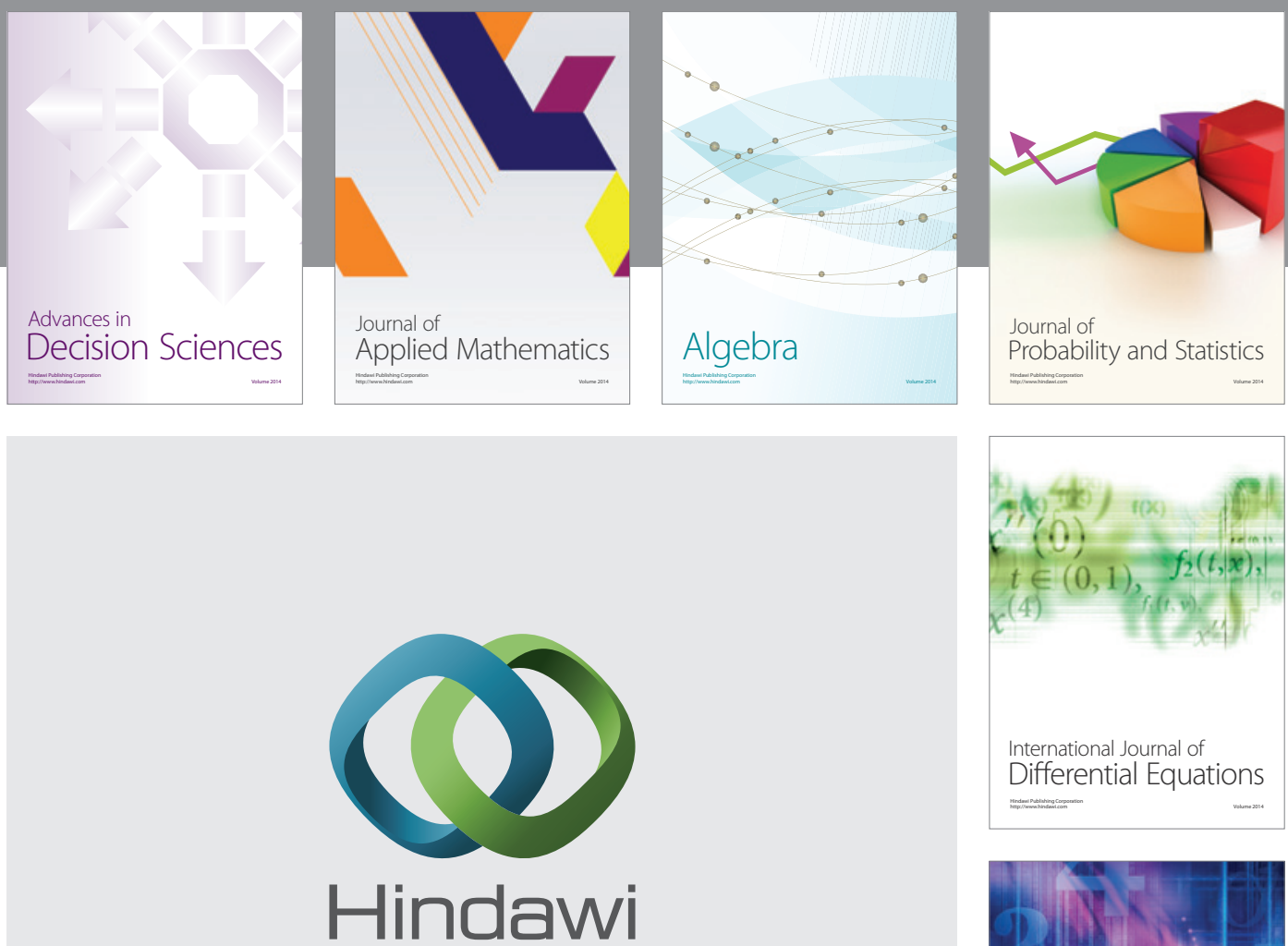

Submit your manuscripts at http://www.hindawi.com
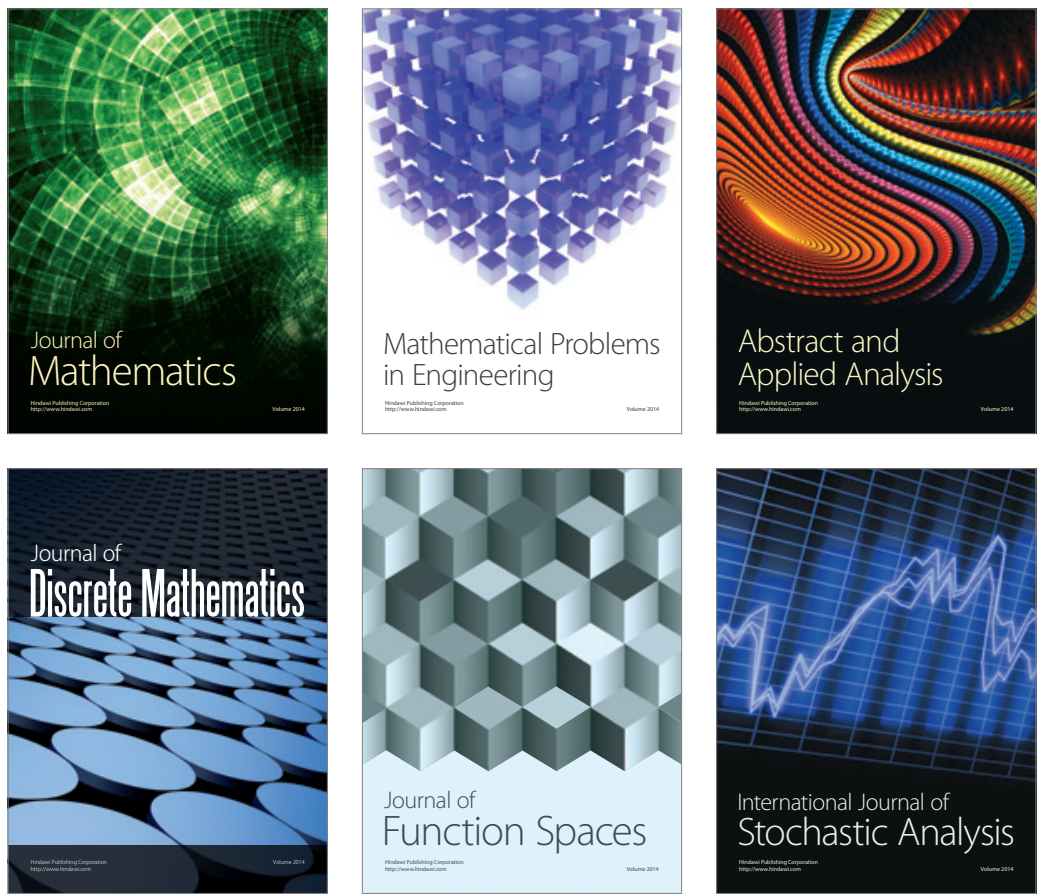

Journal of

Function Spaces

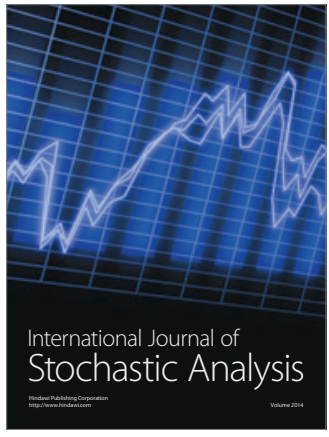

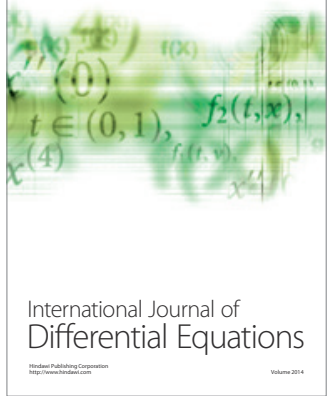
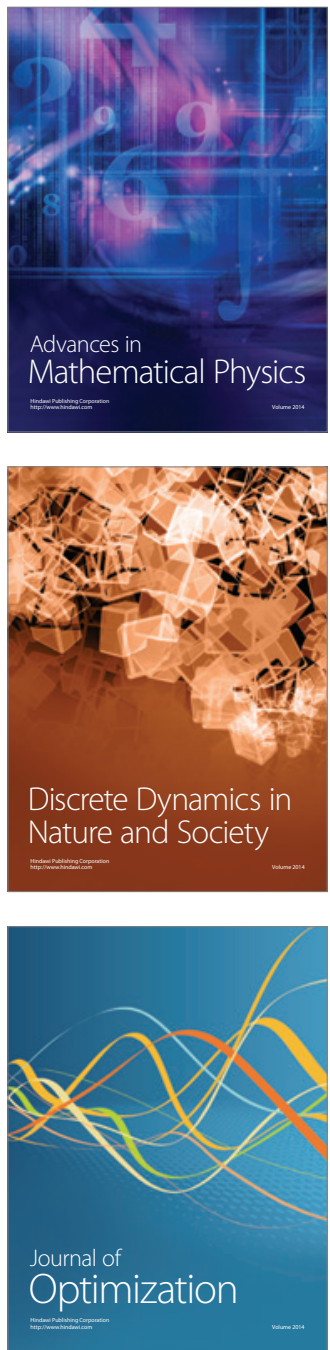